\title{
NEUROLOGICAL CHANGES WITH INTRACAROTID AMYTAL AND MEGIMIDE IN MAN*
}

\author{
BY \\ ROBERT WERMAN, NICHOLAS CHRISTOFF, and PAUL J. ANDERSON \\ From the Laboratory of Electro-oculography, Department of Neurology, Mount Sinai Hospital, New York
}

\begin{abstract}
Alterations in cerebral function produced by intravenously administered drugs are well known. Of these, two classes of drugs are of particular interest in diagnostic neurology: barbiturate and analeptic substances. These drugs have been used to elicit changes in function and neurological defects not otherwise apparent and to help to localize cerebral pathology.
\end{abstract}

The use of drugs, especially "metrazol" (pentamethylenetetrazol), to produce seizures, often focal in the presence of lateralized cerebral pathology, is well documented (Ajmone-Marsan and Ralston, 1957). The pharmacology of the convulsant agents at the synapse may vary greatly but the behavioural effects produced by intravenous administration are phenotypically alike. For example, "metrazol" is an activator of excitatory synapses (Purpura and Grundfest, 1957) while "megimide" (B-B ethyl, methyl glutarimide) is a mild activator of excitatory synapses which exerts its greatest action as a blockader of inhibitory synapses (Purpura, 1958). Despite these differences, both drugs produce similar seizures in the same patient. Patterned focal modes of onset of intravenously produced "metrazol" seizures, with the exceptions of lateralized non-adversive arm jerks and head and eye deviation, localize areas of cerebral dysfunction (Ajmone-Marsan and Ralston, 1957).

Amytal (sodium amobarbital) has been used most widely among the barbiturates in diagnostic neurology. Amytal has been used in eliciting latent organic psychoses (Weinstein, Kahn, Sugarman, and Linn, 1953), sensory defects (Bender and Nathanson, 1950; Jaffe, 1957), and motor deficits (Teng and Bender, 1955). Patients without cerebral disease respond to intravenous "amytal" with nystagmus, dysarthria, ataxia, and sleepiness. The evoked, lateralized findings in neurological patients are always seen against a background of these responses.

While there have been many reports on the effects of the intravenous use of these drugs, behavioural

\footnotetext{
*Supported by the Neurology Research Fund.
}

observations with arterial instillation have been rare. Bennett (1953) injected "metrazol" into the carotid and vertebral arteries of patients with petit mal, producing petit mal states in unanaesthetized patients. Wada (quoted by Roberts, 1958a and b) has used "amytal" and Kjellberg (1959) has used "nembutal" (sodium pentobarbital) in studies on the mechanisms of aphasia produced by carotid injection. These observers find that injection of one side produces aphasia, and injection of the other side does not. Jarvik and Rothballer (1958) reported contralateral circling in cats with "amytal" injected into the carotid, and noted the marked differences between feline and primate carotid artery distributions.

The use of drug injections into the carotid artery permits one to study the pharmacology of a portion of the brain that is relatively restricted by the arterial circulation pattern. Comparison of the results of intravenous and intracarotid injections of a drug should help in localizing the sites of action of the drugs used. However, one must bear in mind the time necessary for circulation to the uninjected hemisphere and basilar system, a period of less than 20 seconds. Observations made before recirculation has occurred are of necessity qualitatively' different from those made after recirculation of the drug. With this limitation in mind, the present study was undertaken.

\section{Material}

Seventeen studies were carried out in 16 patients ranging in age from 17 to 81 years. The patients were undergoing carotid arteriography for a variety of neurological conditions including seizure disorders, migraine, cerebral neoplasms, subarachnoid haemorrhage, post-encephalitic Parkinsonism, and cerebrovascular disorders.

\section{Method}

The patients were all studied after arteriography using "hypaque", when the placing of the needle and circulation patterns could be verified. All 
patients showed filling of the cerebral circulation confined to the ipsilateral hemisphere as far as could be determined by arteriography. The Cournand arterial needle was used to allow for stability of the position of the needle and to minimize discomfort to the patient. Either no premedication or small amounts of atropine were given, The drugs, diluted with normal saline, were rapidly injected in 0.5 to $2.0 \mathrm{ml}$. volumes.

Throughout the procedure the patients were examined by two or more neurologists. At times motion pictures were taken that were subsequently analyzed to confirm behavioural observations. Electroencephalograms (E.E.G.) were recorded throughout on an eight-channel ink-writing electroencephalograph. Communication was evaluated by these tests, namely, naming of objects, counting, comprehension, and spontaneous speech.

Preliminary and control observations were made with saline, "hypaque", "diodrast", atropine, "tensilon", ethanol, "decholin", caffeine, and histamine. No E.E.G. changes were noted with these drugs. Minor and inconsistent behavioural changes were seen including headache (histamine), sleepiness and diffuse paraesthesiae (ethanol), tone changes ("tensilon", atropine), unpleasant taste ("decholin"), and increased pulse rate (atropine). Megimide and "amytal" were administered after these observations. Fourteen of the patients were also studied with intravenous amytal on separate occasions.

\section{Results}

Electro-encephalographic and behavioural changes resulted in all cases after "megimide" and "amytal" injected into the carotid artery. The E.E.G. changes were invariably high-voltage, bilateral slow bursts, at times accompanied by spikes and at times accentuated on the injected side. The E.E.G. changes are reported in detail elsewhere (Werman, Christoff, and Anderson, 1959b). The behavioural effects are the subject of this paper and have already been presented in preliminary form (Werman et al., 1959a).

Megimide.-All patients responded to adequate doses of "megimide" injected into the carotid with motor seizures. The range of effective dosage was surprisingly narrow: all patients responded to $20 \mathrm{mg}$., with a minimal effective dose of $10 \mathrm{mg}$. The average intravenous dose to produce seizures in another and much larger group of patients was between $\mathbf{3 0 0}$ and $\mathbf{4 0 0} \mathrm{mg}$. (unpublished observations, this laboratory). The ratio of the greatest to the least minimum effective seizure dose by the carotid route is $2: 1$ compared with an intravenous ratio of greater than 15:1.
All seizures began focally and showed deviation of the head with or without accompanying movement of the eyes away from the injected side. There was always at least one initial movement of the contralateral arm. Less frequently, twitching of the contralateral face was seen. An adversive onset was seen in one patient. Most of the seizures progressed and became generalized, although they were usually observed to be more violent contralaterally.

None of the patients exhibited unusual seizure patterns. The movements were mostly tonic-clonic, less often somewhat myoclonic. The seizures lasted from 15 seconds to two minutes and all began within five seconds of injection. One seizure remained focal for 45 seconds and never became generalized.

One patient, a woman with grand mal seizures and a left occipital slow-wave E.E.G. focus, was studied on two occasions. Megimide and "amytal" were injected into the left carotid artery during the first procedure and into the right carotid artery the second time. She exhibited mirror image seizures with threshold "megimide" dosage. After the injection of $10 \mathrm{mg}$. "megimide" into the left carotid artery there was a sudden violent jerk of the head to the right. After an appreciable latency, the eyes deviated from the mid-position to the right. First the right arm was extended, then the left. A generalized seizure ensued with apnoea, cyanosis, and clonic movements of all extremities lasting 90 seconds and followed by sleep. Injection of $10 \mathrm{mg}$. "megimide" into the right carotid artery produced a head jerk, deviation of the head to the left with the eyes remaining in mid-position, and a shrill scream. This was followed by extension of the left arm, then the right, generalized clonic movements, apnoea and cyanosis lasting 100 seconds and followed by sleep.

Amytal.-Amytal, 25 to $75 \mathrm{mg}$. injected into the carotid artery, produced behavioural changes in all patients when rapidly injected in 0.5 to $2.0 \mathrm{ml}$. volumes. On several occasions, patients received more than one injection. If separated by sufficient intervals, the results were regularly reproducible. Contralateral hemimotor defects were produced in all patients studied and were always the most striking and profound changes produced. Motor defects always appeared within five seconds and lasted from two to seven minutes and were at all times the last lateralized symptoms to disappear as the patient recovered. The hemiparesis was usually most marked in the arm, the face and leg each decrementally involved. One patient exhibited weakness most marked in the leg after "amytal" injected into the carotid artery of an apparently normal hemisphere. Of the 12 patients without Babinski toe 
signs before intracarotid injection of "amytal", nine developed a contralateral Babinski sign and one developed bilateral Babinski signs. No ocular gaze palsies were observed.

All patients studied were right handed. There were five right carotid injections and 12 left-sided injections. Four of the patients with left carotid injections exhibited mild to moderate aphasic symptoms before injection, none of the right-sided group being aphasic. Following intracarotid injection of "amytal" there was no definite aphasia observed in the non-aphasic patients and no increase in communicative difficulties in the four aphasic subjects. Brief aphonia lasting less than 10 seconds was noted in three non-aphasic patients, two after left carotid injections and one after a right carotid injection. Dysarthria was found in five patients, appearing within 15 seconds in only one. This dysphasic patient was clearly dysarthric within five seconds after the intracarotid injection of "amytal". However, naming of objects, comprehension, and word usage were unaffected. Organic mental changes were not observed in any of the patients after intracarotid injection of "amytal".

The examination routine was directed at the most complete evaluation of language function and motor status possible within 15 seconds. Therefore sensory and field examinations were less complete. Moreover, those patients with sensory or field defects manifested these deficits for periods of less than two minutes. Adequate sensory testing was possible in nine patients, five of whom developed mild to moderate contralateral hemisensory defects, most easily elicitable on double simultaneous stimulation. Visual fields were adequately tested in eight patients, three developing contralateral homonymous hemianopias of moderate degree. The three patients with field defects also had sensory changes.

Nystagmus not present before intracarotid injection of "amytal" appeared in four patients receiving larger amounts of the drug and only after a latency of at least 20 seconds. All but two of the 16 patients studied were examined with intravenous "amytal" administered in increments until sleepiness was reported. Under these conditions, all patients developed nystagmus and dysarthria but in only three were lateralizing signs elicited. In these patients the increase in lateralizing findings was mild and far less dramatic than they exhibited with intracarotid "amytal".

The patient studied bilaterally had no neurological deficit on control examinations and intravenous "amytal" failed to produce lateralizing signs. Injection of $50 \mathrm{mg}$. "amytal" into the left carotid artery produced a mild right hemiparesis with Babinski sign, right-sided extinction and position sense errors, all appearing within five seconds. Neither aphasia nor a field defect could be elicited. The defects could no longer be obtained seven minutes after injection. Injection of $50 \mathrm{mg}$. "amytal" into the right carotid artery was followed within five seconds by a moderate left hemiparesis, a left homonymous hemianopia to double simultaneous stimulation, and poor point localization confined to the left side. There was no aphasia elicitable and all signs cleared in five minutes.

\section{Comment and Discussion}

Our results demonstrate that the effects of "megimide" and "amytal" injected into the carotid artery are different from those found with intravenous injection of these drugs. Although the subjects used displayed a variety of cerebral disorders, the patterns of contralateral onset of seizures with "megimide" and contralateral neurological deficits with "amytal" seemed to be independent of the underlying pathology. Moreover, the one patient studied bilaterally on separate occasions showed profound mirror-image congruence of effects to each of the drugs. The brief latencies and the angiographic check of unilaterality both speak for an ipsilateral hemispheric site of action for these drugs, as do the contralateral behavioural changes. Such variability as is reported can, to a large extent, be explained by nuances in cerebral circulatory patterns from patient to patient.

The lateralized behavioural responses to "amytal" and "megimide" injected into the carotid artery are singular and not reproduced by these drugs injected intravenously. The rapid injection of either drug into the carotid circulation acts on functional groupings in the cerebrum ordinarily not most sensitive to these drugs. The nystagmus, ataxia, dysarthria, and sleepiness seen with intravenous "amytal" are suggestive of brain-stem dysfunction. These responses are not seen with intracarotid administration unless larger doses of "amytal" are used and sufficient time has elapsed for recirculation to the uninjected hemisphere and posterior circulation.

The problem of localization of behavioural activity of a drug such as "amytal" is made difficult by the complex interrelations and functionally overlapping systems of the brain. One must keep in mind the now commonplace observation that in the presence of most lesions of the brain one observes behavioural defects caused not by the lesion but by the reorganization of the intact brain. For example, in the case of "amytal", intravenous administration will eliminate nystagmus present on direct forward gaze and thereby improve visual acuity (Bender, 1946; Nathanson, Bergman, and Bender, 1953). 
This action is surprising in view of the fact that "amytal" consistently produces nystagmus on lateral gaze in normals. In these instances the mechanism is presumably that of two counterbalancing susceptible systems, a lesion of one leading to nystagmus, but both depressed by "amytal".

Our data do not contravert the possible brainstem site of action of intravenous "amytal". Comparing the effects of bilateral simultaneous carotid injections of the barbiturate with those of vertebral injections would be a further aid in determining the threshold sites of action of the drug. These tests would help to discriminate between brain-stem effects and the strong possibility of a complex and diffuse response to intravenous "amytal".

Localization of the sites of action of intravenous "megimide" is even less clear than that for "amytal". The myoclonic jerks and grand mal seizures seen with intravenous "megimide" in patients without focal cerebral disease cannot be localized. It is of interest that the focal behavioural signs which are considered of least value in lateralization with intravenous "metrazol" (Ajmone-Marsan and Ralston, 1957) and probably with intravenous "megimide", viz., non-adversive unilateral arm movements and head and eye deviations, are the very effects that are consistently contralateral with intracarotid "megimide". With intravenous analeptics the onsets of such seizures tend to be unrelated to the localization of pathology, and may vary from side to side in the same patient at different times. No ready explanation for this difference is apparent to us at present.

Bennett (1953) injected "metrazol" both into the carotid arteries and into the vertebral arteries of patients with petit mal seizures. With intracarotid "metrazol" petit mal states always followed but contralateral seizures were seen as well. With vertebral injections, petit mal states were rare and "although long grand mal discharges were frequently elicited, no clonic movements were ever observed". His conclusions on the mechanisms of petit mal disorders are subject to criticism because of (1) the restricted use of petit mal patients, and (2) the technique of repeating increasing doses of "metrazol" until the seizure threshold is reached. Repeated injections during one experiment allow for wide distribution of the drug throughout the brain. Bennett's findings, however, are provocative, suggesting a diffuse or bilateral origin of petit mal, and should be extended in the light of our results.

The success of Wada (quoted by Roberts, 1958a and b) and Kjellberg (1959) in producing aphasia with barbiturates injected into the carotid artery is not supported by our findings, The absence of disturbance in language or ability to communicate in our series was unequivocal in the periods both before and after one circulation time had e'apsed even though all patients developed contralateral neurological defects. It should be noted that intravenous "amytal" has been reported to improve aphasia (Linn and Stein, 1946) but a more detailed study by Bergman and Green (1951) found that intravenous "amytal" had almost no effect on aphasia, increasing the deficit in an occasional patient.

Weinstein et al. (1953) demonstrated that a large percentage of patients with cerebral disorders develop organic mental syndromes with intravenous "amytal". Weinstein and Kahn (1952) further bring to our attention the language disturbance they call "nonaphasic misnaming" (paraphasia). This disturbance of communication, which superficially resembles aphasia, is not lateralizing and is seen only with bilateral cerebral disease and/or diencephalic lesions. Moreover, it is always accompanied by other manifestations of organic psychosis, including those produced by intravenous "amytal". This defect is characterized by misnaming of objects, persons, or places in terms of certain aspects of their function or structure, without other stigmata of aphasia. Roberts (1958b) reports a protocol of an intracarotid "amytal" injection by Wada's technique wherein the patient inititally becomes confused and then develops a "dysphasia", which is not further characterized.

The techniques used by Wada and Kjellberg in eliciting aphasia are different in important details from those used in the present study. Wada (Roberts, $1958 \mathrm{~b}$ ) injects "amytal" in dosages of 175 to $200 \mathrm{mg}$. more than twice the largest dose found necessary in our study to produce easily recognizable neurological symptoms. These large doses are comparable to the dosages of intravenous "amytal" necessary to elicit organic psychosis and other neurological defects in the most sensitive patients. Kjellberg (1959), using "nembutal", instils the drug by slow continuous infusion. In neither study are we told of any precaution to observe the patients before sufficient time has elapsed to distribute the barbiturate via the circulation throughout the brain. Also not indicated is the presence or absence of nystagmus, dysarthria, or other clinical evidence of generalized circulatory dispersion of the barbiturate.

The reported finding by both Wada and Kjellberg that aphasia is produced by their techniques on injection of one side and not the other is an important one. According to the views of Weinstein and Kahn (1955), intracarotid injection of barbiturate, acting on a normal hemisphere, could produce bilateral cerebral dysfunction in the patient whose other (uninjected) hemisphere contains a lesion, thereby causing an organic psychosis and accompanying communicative difficulties. However, this inter- 
pretation does not hold when injection of the diseased hemisphere is the only side to produce aphasia. In such a case, one would like to know if both anterior cerebral arteries are not filled from the same side, a not uncommon situation.

Two further explanations for the discrepancies in the findings on aphasia are available. (1) The threshold for aphasia from barbiturate injected into the carotid artery is considerably higher than thresholds for other lateralizing hemispheric symptoms, and/or (2) aphasia can only be produced by intracarotid barbiturate in the presence of smaller amounts of barbiturates throughout the brain. These interpretations are subject to confirmation by observing (1) the responses to larger doses of barbiturates before one circulation time has elapsed, and (2) the effects of smaller doses in patients premedicated by some other route.

The universal indication for carotid arteriography in the patients used in this study resulted in a highly selected group. However, the diversity of the pathology exhibited and the independence of our results from the underlying disease would partially mitigate the defect of such limited sampling. The diagnostic value of intravenous "amytal" and "megimide" does not appear to be significant. However, the usefulness of intracarotid drug research is attested to by the implications for future study of cerebral physiology and pharmacology.

No serious or persistent morbidity was noted in our study. We feel that judicious administration of intracarotid drugs in humans is a safe procedure and a valuable research method.

\section{Summary}

The behavioural changes after the rapid intracarotid injection of "amytal" and "megimide" are reported. A distinction is drawn between those findings occurring before and those after sufficient time has elapsed to allow recirculation to other parts of the brain.

Seventeen studies on 16 patients are reported, one patient receiving bilateral injections on separate occasions. Although the patients displayed a variety of cerebral lesions, the results were strikingly independent of the location and nature of the disease.
Megimide induced motor seizures most marked contralaterally, with universally contralateral onset.

Amytal produced contralateral motor, sensory, and visual field defects. At no time was aphasia seen. The finding of aphasia by other workers using intracarotid drugs in humans is a safe procedure; effects of the drug are essentially those found with intravenous "amytal".

The sites of action of "megimide" and "amytal" are discussed.

We should like to express our appreciation to Dr. Morris B. Bender, whose advice and encouragement made this study possible.

\section{Addendum}

Since this paper was submitted, a book has appeared with further discussion of the Wada technique (Penfield and Roberts, 1959). On page 98 , we find the following pertinent comment on their results: "The sodium amytal aphasia test . . . has not given clear-cut evidence of bilateral representation of speech but has shown some equivocal results." This statement gains added meaning in the light of our results and discussion.

\section{REFERENCES}

Ajmone-Marsan, C., and Ralston, B. L. (1957). The Epileptic Seizure. Charles C. Thomas, Springfield, III.

Bender, M. B. (1946). Confin. neurol. (Basel), 7, 144.

, and Nathanson, M. (1950). Arch. Neurol. Psychiat. (Chicago) 64, 501 .

Bennett, F. E. (1953). Neurology, 3, 668.

Bergman, P. S.. and Green, M. (195i). Neurology, 1, 471.

Jaffe, R. (1957). Ibid., 7, 531.

Jarvik, M. E., and Rothballer, A. B. (1958). Proc. Amer. Acad. Neurol $10,56$.

Kjellberg, R. N. (1959). Trans. Amer. Neurol. Ass., 1958, p. 76.

Linn, L., and Stein, M. H. (1946). Bull. U.S. Army med. Dept., 5,

Nathanson, M., Bergman, P. S., and Bender, M. B. (1953). A.M.A Arch. Neurol. Psychiat., 69, 427.

Penfield, W., and Roberts, L. (1959). Speech and Brain Mechanisms. Princeton University Press, Princeton, N.J.

Purpura, D. P. (1958). Personal communication.

Purpura, D. P. (1958). H. (1957). J. Neurophysiol., 20, 494.

Roberts, H. L. (1958a). Res. Publ. Ass. nerv. ment. Dis., 36, 449.

- (1958b). A.M.A. Arch. Neurol. Psychiat., 79, 275.

Teng, P., and Bender, M. B. (1955). Neurology, 5, 777.

Weinstein, E. A., and Kahn, R. L. (1952). A.M.A. Arch. Neurol. Psychiat., 67, 72.

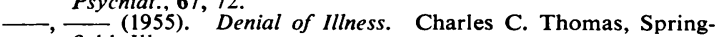
field, IIl. 109,889

Werman, R., Anderson, P. J., and Christoff, N. (1959a). Electroenceph. clin. Neurophysiol., 11, 267.

Christoff, N., and Anderson, P. J. (1959b). Trans. Amer.

Neurol. Ass., 1958, 83rd ann. meeting, p. 74. 